\title{
Abiotic-biotic characterization of Pt/Ir microelectrode arrays in chronic implants
}

\section{Abhishek Prasad ${ }^{1 *}$, Qing-Shan Xue ${ }^{2}$, Robert Dieme ${ }^{3}$, Viswanath Sankar $^{3}$, Roxanne C. Mayrand ${ }^{4}$, Toshikazu Nishida ${ }^{3}$, Wolfgang J. Streit ${ }^{2}$ and Justin C. Sanchez ${ }^{1,4,5}$}

1 Department of Biomedical Engineering, University of Miami, Coral Gables, FL, USA

2 Department of Neuroscience, University of Florida, Gainesville, FL, USA

${ }^{3}$ Department of Electrical and Computer Engineering, University of Florida, Gainesville, FL, USA

${ }^{4}$ Department of Neuroscience, University of Miami, Coral Gables, FL, USA

${ }^{5}$ Miami Project to Cure Paralysis, University of Miami, Miami, FL, USA

\section{Edited by:}

Ulrich G. Hofmann

Albert-Ludwigs-University Freiburg,

Germany

\section{Reviewed by:}

Sofyan H. H. Hammad, Aalborg

University, Denmark

Susanne Löffler, Karolinska Institute, Sweden

\section{${ }^{*}$ Correspondence:}

Abhishek Prasad, Department of Biomedical Engineering, B91

University of Miami, 1251 Memorial Drive, McArthur Engineering Annex \#219A, Coral Gables, FL 33146, USA e-mail:a.prasad@miami.edu
$\mathrm{Pt} / \mathrm{Ir}$ electrodes have been extensively used in neurophysiology research in recent years as they provide a more inert recording surface as compared to tungsten or stainless steel. While floating microelectrode arrays (FMA) consisting of $\mathrm{Pt} / \mathrm{lr}$ electrodes are an option for neuroprosthetic applications, long-term in vivo functional performance characterization of these FMAs is lacking. In this study, we have performed comprehensive abiotic-biotic characterization of $\mathrm{Pt} / \mathrm{lr}$ arrays in 12 rats with implant periods ranging from 1 week up to 6 months. Each of the FMAs consisted of 16-channel, $1.5 \mathrm{~mm}$ long, and $75 \mu \mathrm{m}$ diameter microwires with tapered tips that were implanted into the somatosensory cortex. Abiotic characterization included (1) pre-implant and post-explant scanning electron microscopy (SEM) to study recording site changes, insulation delamination and cracking, and (2) chronic in vivo electrode impedance spectroscopy. Biotic characterization included study of microglial responses using a panel of antibodies, such as Iba1, ED1, and anti-ferritin, the latter being indicative of blood-brain barrier (BBB) disruption. Significant structural variation was observed pre-implantation among the arrays in the form of irregular insulation, cracks in insulation/recording surface, and insulation delamination. We observed delamination and cracking of insulation in almost all electrodes post-implantation. These changes altered the electrochemical surface area of the electrodes and resulted in declining impedance over the long-term due to formation of electrical leakage pathways. In general, the decline in impedance corresponded with poor electrode functional performance, which was quantified via electrode yield. Our abiotic results suggest that manufacturing variability and insulation material as an important factor contributing to electrode failure. Biotic results show that electrode performance was not correlated with microglial activation (neuroinflammation) as we were able to observe poor performance in the absence of neuroinflammation, as well as good performance in the presence of neuroinflammation. One biotic change that correlated well with poor electrode performance was intraparenchymal bleeding, which was evident macroscopically in some rats and presented microscopically by intense ferritin immunoreactivity in microglia/macrophages. Thus, we currently consider intraparenchymal bleeding, suboptimal electrode fabrication, and insulation delamination as the major factors contributing toward electrode failure.

Keywords: Pt/Ir microelectrodes, neural interface, blood brain barrier (BBB), neuroinflammation, abiotic, biotic, floating microelectrode arrays (FMA), impedance

\section{INTRODUCTION}

The development of clinically viable microelectrode arrays for humans has produced multiple engineering design and neurophysiological requirements that are believed to be necessary for facilitating high performance (Jackson and Zimmermann, 2012). These include the ability to target and access the activity in ensembles of neurons located in cortical and deep brain structures for treating a variety of neurological problems including paralysis (Ethier et al., 2012; Hochberg et al., 2012; Collinger et al., 2013), stroke (Iosa et al., 2012), movement disorders (Andrews, 2010;
Rouse et al., 2011), epilepsy (Morrell, 2011; Truccolo et al., 2011), and neuropsychiatric disorders (Maling et al., 2012). Not only is short-term access of these neurons important but also the microelectrode arrays should be able to sense and stimulate targeted neurons reliably, which could be of the order of tens of years. From a materials science perspective, there are differences in the design considerations between sensing and stimulation but in general, it is desirable to have stable electrodes that do not corrode (Sanchez et al., 2006; Prasad et al., 2012), produce stable impedance (Ward et al., 2009; Prasad and Sanchez, 2012), and are 
robust after repeated delivery of current through them (Johnson et al., 2005; Otto et al., 2005, 2006; Koivuniemi and Otto, 2011; Lempka et al., 2011). A variety of materials including tungsten, platinum, platinum-iridium, and iridium oxide coatings have been used to improve the reliability of recordings (Geddes and Roeder, 2003; Cogan, 2008; Ward et al., 2009). Closely coupled to the abiotic materials aspects are the biotic responses to chronically implanted microelectrode arrays. The biotic effects include the disruption of the blood-brain barrier (BBB) (Prasad et al., 2011; Karumbaiah et al., 2013; Saxena et al., 2013), tissue inflammatory response involving astroglial and microglial reactions occurring at/around the implanted site, and macrophage recruitment to the implanted site (Schmidt et al., 1976; Stensaas and Stensaas, 1978; Edell et al., 1992; Kam et al., 1999; Turner et al., 1999; Szarowski et al., 2003; Biran et al., 2005; Lee et al., 2005; Polikov et al., 2005; Biran et al., 2007; McConnell et al., 2009b; Winslow and Tresco, 2010; Thelin et al., 2011; Prasad et al., 2012). The abiotic effects include the changes occurring at the electrode recording sites such as corrosion and insulation delamination and cracking that alters the electrochemical properties of the electrode recording surface area (Geddes and Roeder, 2003; Patrick et al., 2011; Prasad and Sanchez, 2012; Prasad et al., 2012; Streit et al., 2012; Kane et al., 2013). Both these effects are dynamic in nature, occur concurrently, and cannot be isolated from one another. Therefore, high-performance arrays should produce minimal tissue reactivity including disruption of the blood-brain-barrier (Prasad et al., 2012), glial response (Frampton et al., 2010; Winslow and Tresco, 2010; Prasad et al., 2012), and neuronal damage (McConnell et al., 2009b). Finding the optimal balance of all of these design considerations is challenging and various electrode tip geometries (Andrei et al., 2012) and multiple array solutions have been proposed including microwire arrays, floating planar silicon arrays, floating 2-D silicon arrays, and floating microwire arrays (Drake et al., 1988; Rousche and Normann, 1998; Williams et al., 1999a,b; Rousche et al., 2001; Csicsvari et al., 2003; Kipke et al., 2003; Nicolelis et al., 2003; Vetter et al., 2004; Patrick et al., 2006; Jackson and Fetz, 2007; Musallam et al., 2007; Kozai et al., 2012a; Kim et al., 2013; Richter et al., 2013). Unique among these available solutions is the "floating" aspect of the array design. It is believed that floating arrays can mitigate many of the challenges of chronic implantation as they have been shown to cause reduced lesions due to electrode array micro-movements in the brain tissue (Biran et al., 2007). Systematic and quantitative study of each of these array types is necessary to prove their benefits and weaknesses in the context of electrode performance and failure.

In this work, floating microelectrode arrays (FMA) fabricated by Microprobes (Gaithersburg, MD) are studied. These electrodes are made of $\mathrm{Pt} / \mathrm{Ir}$ which provide a more stable and inert electroderecording site that is less prone to corrosion and surface changes compared to other metals such as tungsten and stainless steel (Geddes and Roeder, 2003; Cogan, 2008; Patrick et al., 2011; Prasad et al., 2012; Kane et al., 2013). In addition, they are laser cut with a conical tip to reduce the trauma during insertion. Unlike the Utah array, which is the only other commercial design that is similar, the FMA can be custom cut for both deep brain and cortical targets. The arrays are fabricated on a planar substrate and connected via a thin flexible cable, which allows the array to float with the brain. While FMAs have been used in a variety of cortical and spinal applications for basic neurophysiological and neuroprosthetic applications, they have not undergone comprehensive characterization to quantify their electrophysiological properties over long-durations (weeks to months) in vivo. We have recently developed an in vivo testbed (Prasad and Sanchez, 2012; Prasad et al., 2012; Streit et al., 2012) to quantify the failure mechanisms of microelectrodes and have shown that there are a variety of biotic and abiotic aspects that contribute to electrode performance. Since microelectrode array design is so diverse among the available options (Utah, FMA, microwires, Michigan), each design needs to be evaluated independently using a common set of relevant metrics. Toward developing a comprehensive characterization of FMA performance and failure, this study performs an in vivo long-term abiotic and biotic analysis with an aim to build a quality control system for microelectrodes. FMAs were implanted into the rat somatosensory cortex for implant duration ranging from 7 days up to 6 months. Abiotic analysis included (1) pre-implant and post-explant SEM imaging for qualitative comparison of the changes that occur at the electrode recording surface, and (2) chronic in vivo impedance spectroscopy for studying the changes in the electrode impedance chronically. Biotic analysis was assessed using microglial activation and degeneration and BBB disruption. Chronic electrode performance was quantified via array yield. The array yield, specified by the number of recording sites producing single neuron recordings, provides insight to the overall health of the array. High-performance electrode arrays produce single neuron recordings from a maximal number of recording sites. Likewise, failing electrode arrays produce low yields. It has been shown that impedance spectroscopy provides insight to the fundamental aspects of biotic and abiotic interactions that affect array performance (Grill and Mortimer, 1994; Merrill and Tresco, 2005; Ludwig et al., 2006, 2011; Williams et al., 2007; McConnell et al., 2009a; Karumbaiah et al., 2013). Combining standard electrophysiological measurements with abiotic and biotic analysis can produce comprehensive insight to electrode failure.

\section{METHODS}

All procedures were approved by the University of Miami Institutional Animal Care and Use Committee (IACUC). Twelve age-matched adult male Sprague-Dawley rats, all weighing between 300 and 350 grams (at implant time) were used in this study. Animals were divided into short-term implant group ( $\leq 2$ weeks) (Turner et al., 1999; Winslow and Tresco, 2010) and chronic groups ( $\leq 6$ months) (Williams et al., 1999b; Polikov et al., 2005; Winslow and Tresco, 2010). In this study, 3 animals were implanted for up to 7 days, 2 for 15 days, 1 for 3 weeks, 3 for up to 3 months, and 3 for 6 months, respectively. The 7-days and 15-days animals were classified in the short-term implant group whereas the remaining animals were classified in the chronic group. All animals were euthanized at the end of their prospective study period intentionally and the brain tissue was harvested for histological analysis. Table 1 summarizes the pre-implant and post-explant SEM observations, functional performance, and histopathological summary for all 12 animals included in the study. 


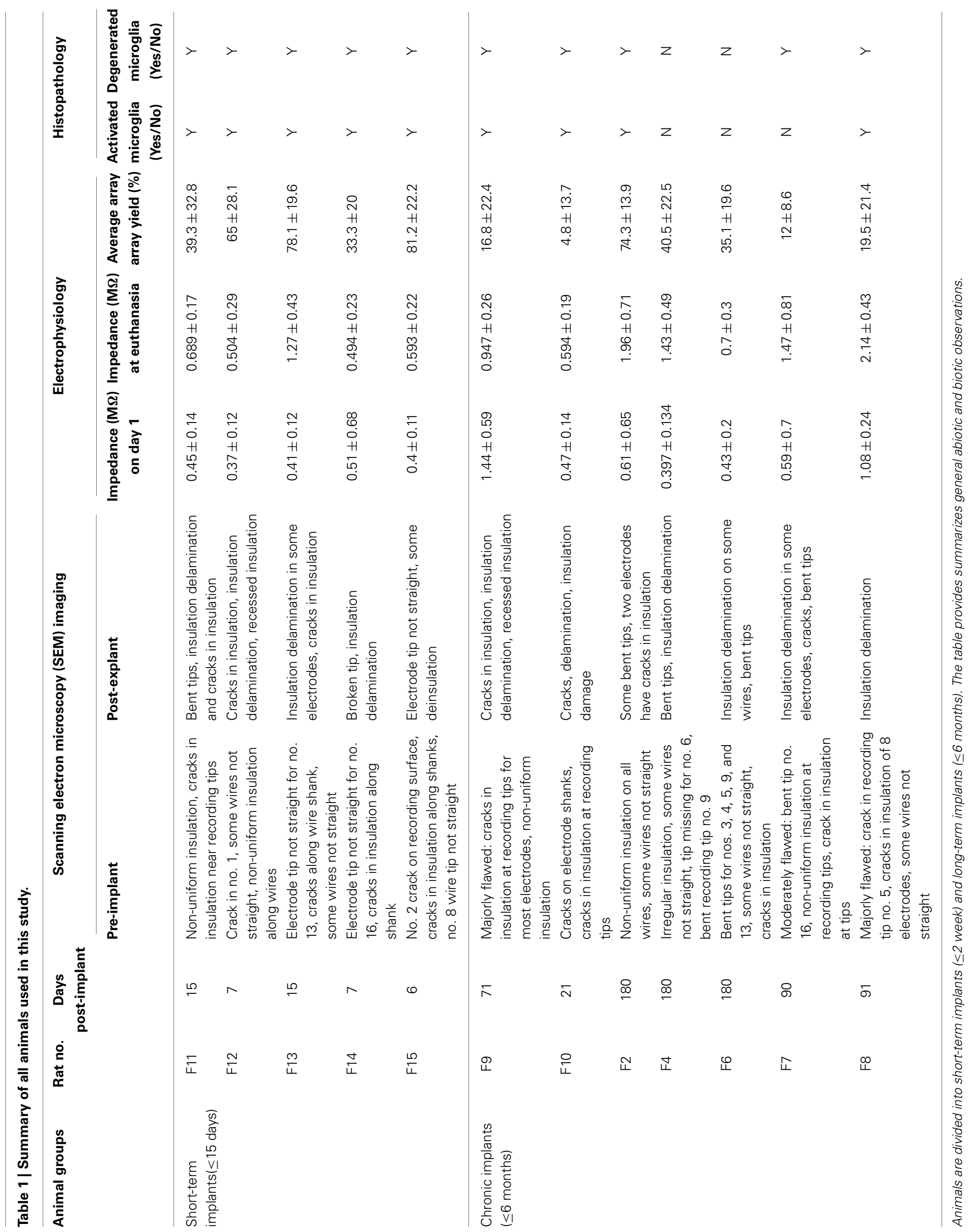




\section{ELECTRODE}

We characterized 16-channel FMA (MicroProbes, Inc, MD) for our implants in all animals (Musallam et al., 2007; Mollazadeh et al., 2011). The microelectrode array consisted of 16 microwires [Pt/Ir (70/30\%) $75 \mu \mathrm{m}$ diameter at the base which tapered to a fine tip] attached to a ceramic substrate (Figure 1A). Each of the microwires were $1.5 \mathrm{~mm}$ long and separated by $400 \mu \mathrm{m}$. The microwires were insulated with parylene-C, the thickness of which was $3 \mu \mathrm{m}$ along the wire shaft and the microwires were laser cut at the tip, which produced a conical recording surface. The microwires were soldered to gold wires, which were bundled together and soldered to a 16-channel Omnetics connector that was fixed on the skull. The wire bundle was coated with medical grade silicone to provide insulation.

\section{SCANNING ELECTRON MICROSCOPY (SEM) IMAGING}

In order to evaluate the structural changes in chronically implanted microelectrode arrays, all arrays were imaged before implantation and after explantation using a variable pressure scanning electron microscope (Hitachi S-3000N VP-SEM). The environmental mode in the VP-SEM was chosen for imaging the arrays to enable direct placement of the samples into the SEM chamber without the use of carbon or conductive coating. This is very critical especially for pristine electrodes as they will be implanted into an animal after the SEM imaging procedure. Such a method was used so as to cause no damage to the arrays from the imaging process for both pre-implant as well as explanted arrays. When the pre-implant images were taken, electrode arrays were handled with care to prevent any damage to the microwires. The post-explant images were taken on the electrode arrays extracted from the implanted tissue. The post-explant arrays were carefully placed on a holder positioned on to the SEM stage. The following parameters were used to take the pre- and post-implant images: (1) environmental secondary electron detector (ESED) mode with an acceleration potential of $12 \mathrm{kV}$, (2) working distance range was set between 18 and $40 \mathrm{~mm}$, and (3) magnification was varied according to need and the minimum magnification was set at $20 \mathrm{X}$.

\section{IMPEDANCE TESTING PROCEDURE}

Electrode impedance spectroscopy was performed using NanoZ (TDT, Alachua, FL) using procedures described in detail elsewhere (Prasad and Sanchez, 2012). Briefly, NanoZ measures impedance by applying a small sinusoidal voltage of $4 \mathrm{mVpp}$ at specific frequencies of $1 \mathrm{~Hz}, 2 \mathrm{~Hz}, 5 \mathrm{~Hz}, 10 \mathrm{~Hz}, 20 \mathrm{~Hz}, 50 \mathrm{~Hz}$, $100 \mathrm{~Hz}, 200 \mathrm{~Hz}, 500 \mathrm{~Hz}, 1 \mathrm{kHz}$, and $2 \mathrm{kHz}$, respectively. The small amount of voltage applied by NanoZ causes a maximum test current of $1.4 \mathrm{nA}$ RMS to flow through the measuring circuit. The impedance measurement protocol was approved by the electrode manufacturer. We combined repeated impedance measurements following the above protocol with scanning electron microscopy (SEM) imaging to verify that electrode recording surfaces were not altered due to the measurement process itself. Impedance spectroscopy in vitro was performed for electrode arrays with respect to a low impedance stainless steel reference wire prior to implantation in $0.9 \%$ phosphate buffered saline (PBS) for obtaining baseline values for each array. The two-electrode method has been deemed suitable for measuring microelectrode impedance owing to the large difference in impedance between the electrode being tested and the distant low impedance reference wire (Brett and Brett, 1993; Geddes, 1997; Williams et al., 2007). In vivo measurements were made with respect to the same stainless steel wire tied to the skull screw while the animals were lightly anesthetized during impedance measurements. In vivo, impedance was measured 3-4 times a week before electrophysiological recordings. In our testing, the NanoZ calculated the impedance values for each electrode by repeating the process 20 times and reported the average of those values to reduce errors due to measurement.

\section{SURGICAL PROCEDURE}

Electrodes were individually sterilized using ethylene oxide gas prior to implantation and sterile surgical techniques were followed for all implant surgeries. Animals were anesthetized with an induction of isoflurane $(4 \%)$ and $\mathrm{O}_{2}(2 \mathrm{~L} / \mathrm{min})$ and deep anesthesia level was maintained for the entire duration of the surgical procedure using isoflurane $(1.5 \%)$ and $1 \mathrm{~L} / \mathrm{min}_{2}$. Xylazine (5 mg/kg, subcutaneous) was used to maintain an even plane of anesthesia and as a muscle relaxant. Upon fixing the animal's head in a stereotaxic frame (Kopf Instruments, Tujunga, CA) to stabilize the skull, a midline incision was made on the skin from between the eyes up to the ears and the periosteum was scraped from on top of the skull. The skull surface was cleaned and the bregma and lambda landmarks were identified. Four stainless steel screws were manually drilled into the skull. Using stereotaxic coordinates, the craniotomy location $(1 \mathrm{~mm}$ lateral and $1.8 \mathrm{~mm}$ caudal to the bregma) was marked which correspond to the somatosensory cortex. A $5 \mathrm{~mm}$ diameter circular craniotomy was drilled at the above marked location (Figure 1B) and the dura was then cut using microscissors to expose the cortical surface. The setup shown in Figure 1C was used to hold the electrode connector and position the electrode array using a vacuum pump wand. The electrode array was slowly $(\sim 0.1 \mathrm{~mm} / \mathrm{min})$ lowered into the cortex using the micropositioner (Kopf Instruments, Tujunga, CA). Slow speed of insertion was used for these FMA as recommended by the manufacturer and similar to studies that have shown success in recording single neurons with a slow insertion speed for microwire arrays (Nicolelis, 1999). A small piece of gelfoam soaked in sterile saline was used to cover the craniotomy and dental acrylic (A-M Systems, Carlsborg, CA) was placed on and around the craniotomy. Extreme care was taken such that none of the acrylic entered the craniotomy. The ground and reference wires were tied to the skull screw and the entire area was filled with acrylic. Animals were placed under supervision in a recovery box kept on a heating pad post-surgery and housed individually upon recovery. Bupivacaine was used around the wound site after the surgery and Carprofen $(5 \mathrm{mg} / \mathrm{kg}$, subcutaneous) was used as the analgesic. No antibiotics or any other drugs were used in this study.

\section{RECORDING PROCEDURE}

Animals were recorded 3-4 times each week. Electrode impedance spectroscopy was performed prior to each recording session. The animal was connected to a preamplifier that was optically isolated from a real-time digital signal processor (RZ2, TDT, FL). 

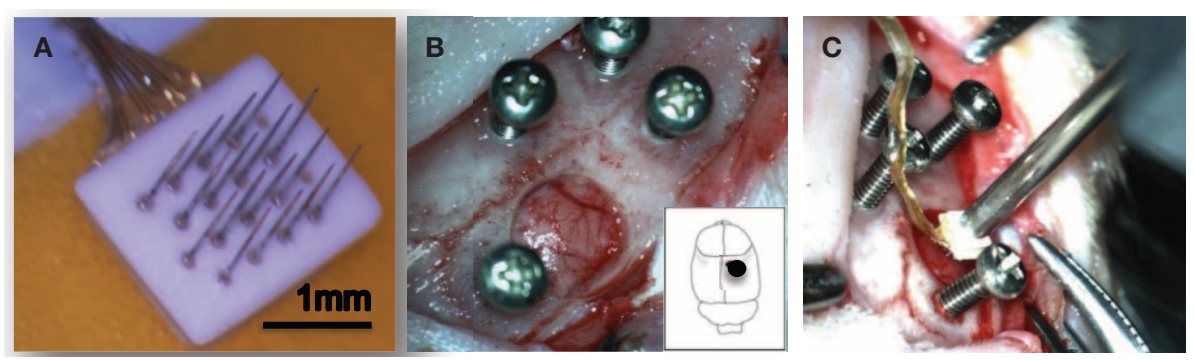

FIGURE 1 | Experimental setup. A 16-channel Pt//r floating microelectrode array (FMA) consisting of $1.5 \mathrm{~mm}$ long and $75 \mu \mathrm{m}$ diameter with tapered microwires tips was used for all implants (A). Surgical implantation procedure in the rat somatosensory cortex is shown (B,C).

A custom made program ( $\mathrm{RPvDx}, \mathrm{TDT}, \mathrm{FL})$ was used to acquire neural signals sampled at $24414.06 \mathrm{~Hz}$. Signals were band-pass filtered between 0.5 and $6 \mathrm{kHz}$ and online spike sorting based on boxes and thresholding as determined by the experimenter was used to isolate neuronal waveforms. Offline spike sorting was then performed to verify isolated single units and remove other artifacts. Waveforms were classified as single units based upon the repeatability of waveform shape and peak-to-peak amplitude with respect to the background noise (Suner et al., 2005). Electrophysiological recordings were quantified via array yield, which was defined as the percentage of electrodes within an array that were able to isolate at least one neuron during a recording session. Array yield was used as a functional measure for electrode assessment. An electrode was said to isolate a neuron (single unit) when at least 100 reproducible biphasic waveforms could be collected during a session.

Signal-to-Noise ratio (SNR) was calculated using the formula: $S N R=\frac{V}{2 * \sigma}$, where $V$ is the peak-to-peak amplitude of the mean waveform and $\sigma$ is the standard deviation of the noise. These methods have been described in detail elsewhere (Suner et al., 2005). Figure 2A provides a general measure of the signal quality where offline sorted waveforms from four isolated neurons is shown with SNR value calculated for each neuron. The neuron waveforms shown are for animal F6 and are from different electrodes and different sessions. All waveforms were collected and shown within a $1.2 \mathrm{~ms}$ time-window. Weekly signal-to-noise levels are shown for four animals (F6-9) during their respective implant durations in Figure 2B. In general, SNR values ranged between 3 and 6 and were comparable to those reported earlier by other studies (Suner et al., 2005; Ward et al., 2009). We found that SNR values were not dependent upon implant durations or daily changes in impedance values and also not correlated to the electrode performance.

\section{IMMUNOHISTOCHEMICAL PROCEDURES}

Immunohistochemical procedures have been described in detail elsewhere (Prasad et al., 2012). Briefly, microglial cells in brain sections were examined immunohistochemically using the rabbit polyclonal primary antibody Ibal (Wako, 019-19741, diluted at 1:800). Phagocytic microglia were identified using ED1 antibody (mouse anti-rat monocytes/macrophages (CD68), Chemicon; MAB1435, diluted at 1:300). Immunolabeling for the iron storage protein was performed using antiferritin polyclonal antibody (rabbit anti-horse spleen ferritin, Sigma, F6136, diluted at 1:800). Ferritin, an iron storage protein that becomes expressed in some microglia in the human brain are often dystrophic rather than activated (Dijkstra et al., 1985). Ferritin staining of microglia seems to be induced under certain injury/disease conditions when the blood-brain barrier is disturbed and there is a need for iron sequestration. The ferritin antibody also binds to oligodendrocytes in the normal uninjured central nervous system (Lopes et al., 2008). Quantitative analysis of antigen expression was conducted on images using Image J program $(\mathrm{NIH}$, Bethesda, MD). The total positive area of an antigen expression per unit section area (i.e., per microscopic field $\times 20$; total area size $267320.12 \mu \mathrm{m}^{2}$ ) were scored in an image captured from each side of rat brain by using Image J program. The mean values and standard deviation of the counts were computed. An intact microglial cell was defined as a cell with a cell body area larger than $30 \mu \mathrm{m}^{2}$ and less than $300 \mu \mathrm{m}^{2}$ for Ibal and ferritin labeling. The ED1 positive particle was defined as areas from 1 to $10 \mu \mathrm{m}^{2}$. The difference between the electrode implant side and the contralateral side was interpreted to reflect an approximation of microglial cell function.

\section{RESULTS \\ SEM IMAGING OBSERVATIONS}

A comparison of the pre-implant and post-explant SEM images of the electrode arrays was made to analyze qualitatively the changes at the electrode recording surface morphology for all animals. All electrodes prior to implantation were imaged to observe any pre-implant defects in the electrodes due to the manufacturing process. We observed several defects occurring as a result of manufacturing most commonly present in the recording surfaces and the interface between the recording surface and the insulation. The manufacturing defects most commonly present were cracks in the insulation material, non-uniform insulation, bent and broken recording tips, cracks on the recording tips, and non-uniform deinsulation (Figure 3). Figure 3 shows examples of electrodes with pre-implant manufacturing imperfections for 4 of the longest term animals (3-6 months implant period). These images were taken prior to electrode implants. Note that not all the electrodes within an array had issues but approximately $25 \%$ or more electrodes tend to have one or the other issue as described above. Thus, there was a large variation between electrodes within an array with some electrodes with no issues whereas others with 

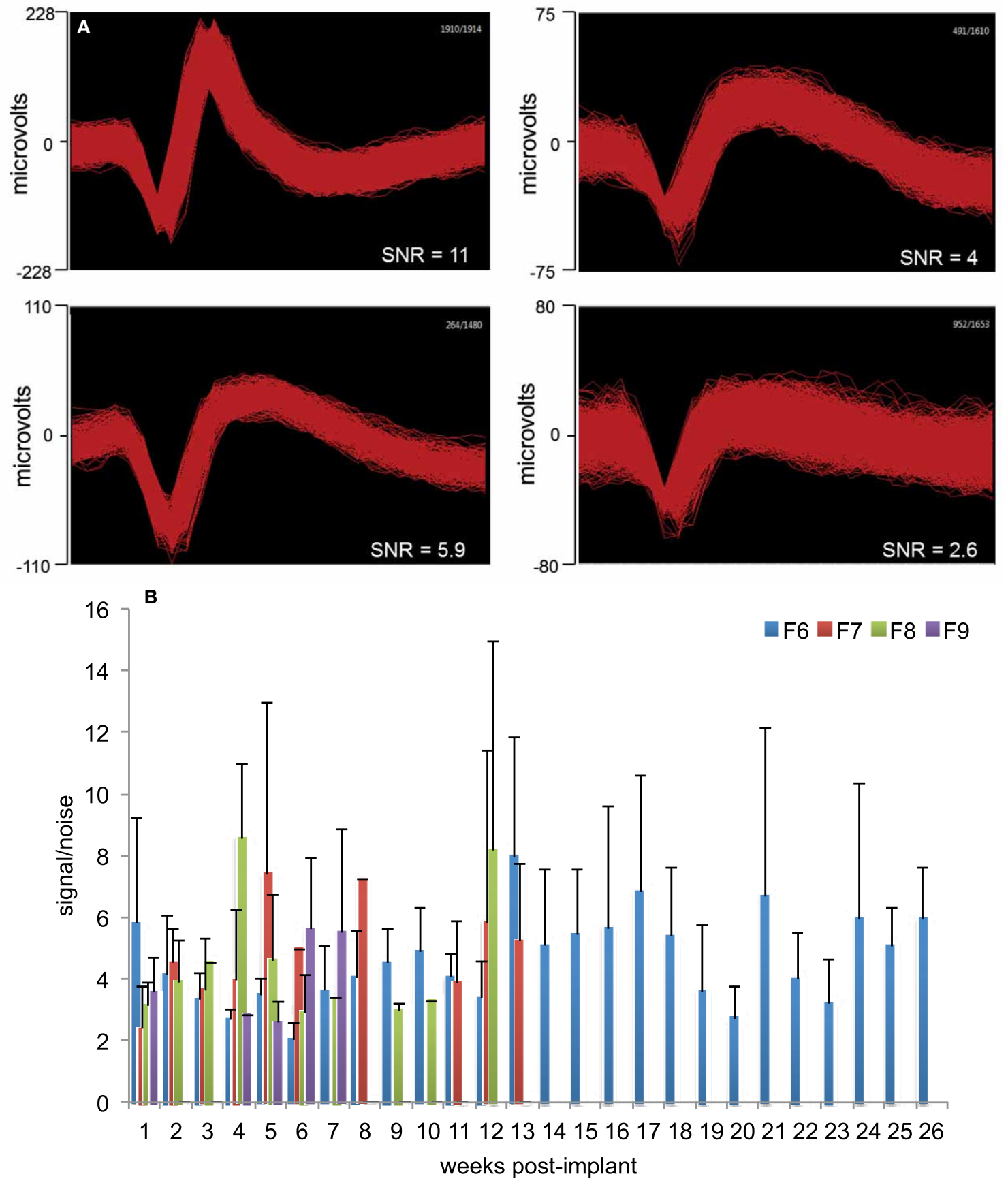

FIGURE 2 | Signal quality and SNR. (A) Representative offline sorted waveforms isolated from individual electrodes to depict signal quality. High (>5), medium (3-5), and low SNR (<3) waveforms are shown. In general, SNR values were in the range 3-6. Waveforms are shown within a $1.2 \mathrm{~ms}$ time-window. (B) Weekly signal-to-noise levels for animals F6, F7, F8, and F9 are shown. SNR values were comparable to those reported earlier by other studies. SNR values were not dependent upon implant durations or daily changes in impedance values. pre-implant imperfections. Pre-implant and post-explant SEM images were taken for qualitative comparison of the changes that occur at the electrode recording surface and insulation material. Figure 4A shows such changes occurring after chronic implantation in animals where the electrodes underwent moderate to minimal changes at the recording surfaces. We observed that $\mathrm{Pt} / \mathrm{Ir}$ undergoes little corrosion for implants up to 6 months. However, there was a great variability among individual electrodes even within the same array with regard to corrosion and/or insulation deterioration. While electrode corrosion did not appear to be a significant problem with $\mathrm{Pt} / \mathrm{Ir}$, we observed severe delamination and cracking of insulation material. Gross morphological 


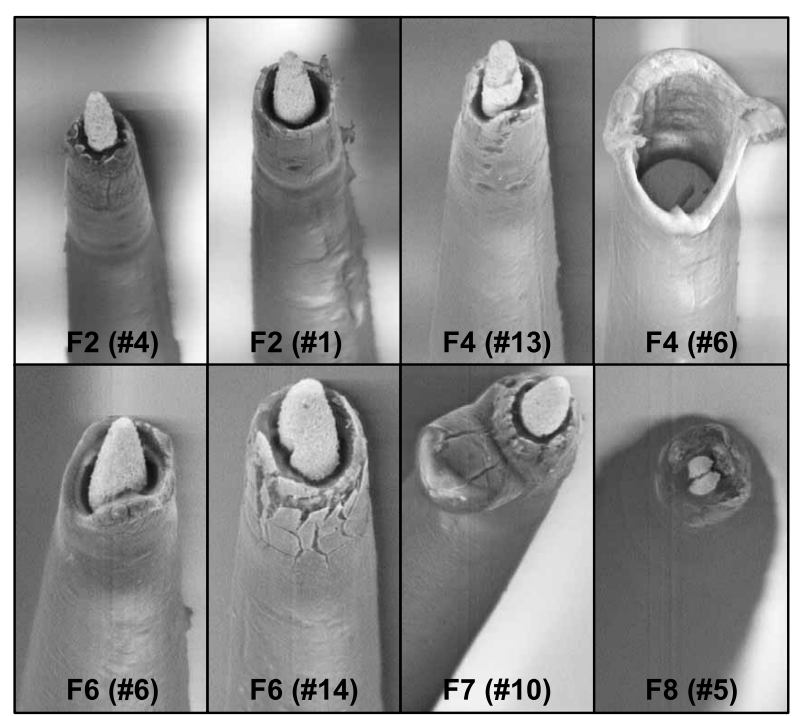

FIGURE 3 | Manufacturing variability. We observed large variation between electrodes within the same Pt/Ir array. Shown here are 8 wires from four arrays pre-implant with imperfections as a result of manufacturing process. One of the wires is missing the recording tip whereas others have cracks in the insulation/recording tip, and uneven insulation at the interface of the recording surface/insulation.

changes occurred at the recording surface in the form of bent tips, cracks appearing in the insulation material, and insulation delamination. Figure 4B show 12 representative electrodes from 7 long-term animals (3-6 months) where severe damage to the insulation material was observed. Insulation damage and delamination resulted in a decrease in electrode impedance during the chronic lifetime and affected functional performance (Figure 5). Figure 5 shows such an example for 4 electrodes from 3 longterm animals (F4 and F6: 6 months; F9: 71 days) where electrode impedance declined during the latter part of the implant period. Interestingly, as the electrode was undergoing these morphological changes, the functional performance (electrode yield) became poor.

\section{IMPEDANCE AND ELECTROPHYSIOLOGICAL OBSERVATIONS}

To study general trends in impedance magnitude, $1 \mathrm{kHz}$ impedance was chosen throughout this study as this is the fundamental frequency of an action potential and the value commonly used to report impedance in other studies (Ward et al., 2009). Figure 6 shows the $1 \mathrm{kHz}$ impedance trends and the array yield during the implant period for 4 long-term animals (F2, F4, F6: 6 months; F8: 3 months). The figure plots the average array yield (red bars) and average impedance (blue trace) from 16 electrodes on each of the recording days. Impedance values increased progressively to 2-3 times the post-implant values by the 1-week period. In general, impedance values continued to increase until the 3-4 week period with values in the range 1.5-2.5 $\mathrm{M} \Omega$ after which they decreased. There was also greater variation in the daily impedance values as suggested by the large error bars. The impedance values generally decreased after the 15-day period with occasional fluctuations in the chronic period thereafter. These general impedance magnitude trends with large daily variations were consistent among all animals. Array yield generally was high post-implantation and declined in the days following surgery. In general, an inverse relationship between the daily impedance measurements and the array yield was observed for all our long-term animals.

\section{HISTOPATHOLOGY OBSERVATIONS}

Light microscopic examination of sections stained with microglial markers revealed a rather heterogeneous picture of histopathological changes (Figure 7), but some clear trends were apparent. In general microglial activation, as judged by cell hypertrophy and cell density on Iba1-stained preparations was strongest during early survival times and tended to decrease with increasing survival post-implantation, that is the intensity of neuroinflammation declined over time. Expression of ED1 antigen, indicative of ongoing phagocytic activity, was variable and showed no apparent correlation with post-implant survival times. The ED1 antigen was always present in microglia/macrophages. Expression of ferritin was not related to post-implantation survival times but instead was variable and likely related directly to the extent of vascular damage and hemorrhage (Figure 8). Dense encapsulation of electrodes was not observed in many of the animals and at most involved two cell layers surrounding electrode tracks (Table 2). The extent of encapsulation was not correlated with electrode performance, e.g., F8 (Figure 7; Table 2).

\section{COMBINED ABIOTIC-BIOTIC ANALYSIS}

One or more metrics can be used for predicting electrode failure through modeling approaches that would provide us with a reliable estimate of electrode failure. A combined analysis that included histopathological and morphological evaluation combined with functional assessment of these arrays was performed to support our hypothesis of the various abiotic and biotic failure modes that contributes to electrode failure (Figure 9). All histopathological markers (Iba1, ED1, and Ferritin) were normalized across all animals for comparison. We used the following measures to define functional performance: poor ( $<25 \%$ array yield), moderate ( $25-70 \%$ array yield), and good ( $>70 \%$ array yield). All the longest term animals (6-months implant: F2, F4, F6) had moderate to good performance with low histopathology marker levels. Among animals that had good performance, 4 out of 7 animals had very low levels of ferritin expression. The animals (F11, F12, F15) which had higher ferritin levels were very short-term implants. It is likely that ferritin along with other markers were upregulated due to surgical trauma. Even then, the ferritin levels in these animals were much lower than other animals (F7, F9, F10). Therefore, low levels of ferritin was correlated with better functional performance. Electrodes that were poorly manufactured to begin with underwent gross morphological changes overall and had poor functional performance (F7, F8, F9, F10). This supports our hypothesis that insulation delamination/cracking and manufacturing variability leads to reduced impedance over time and degradation in functional performance. There were animals (F11, F12, F15) that had good performance with high 
A
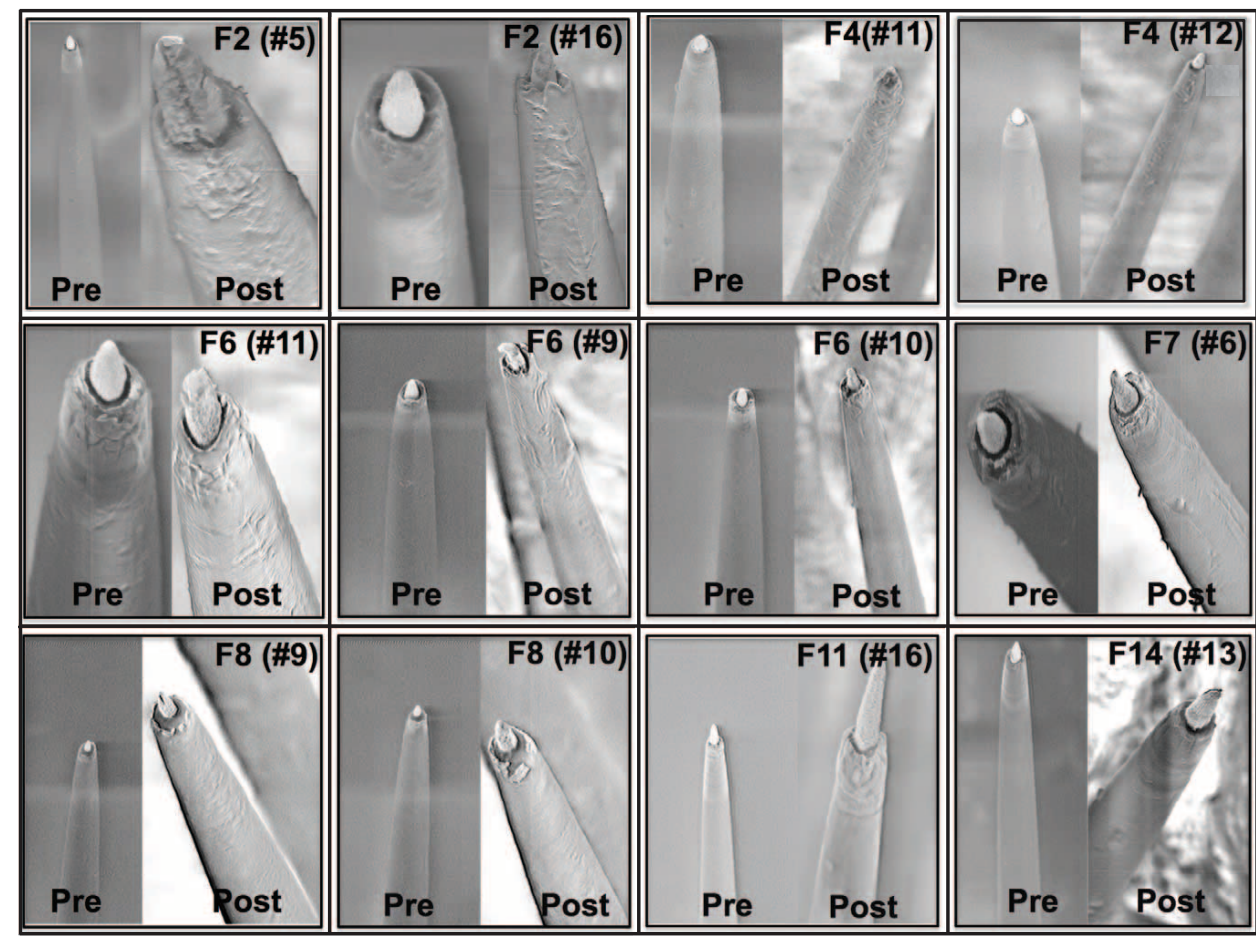

B

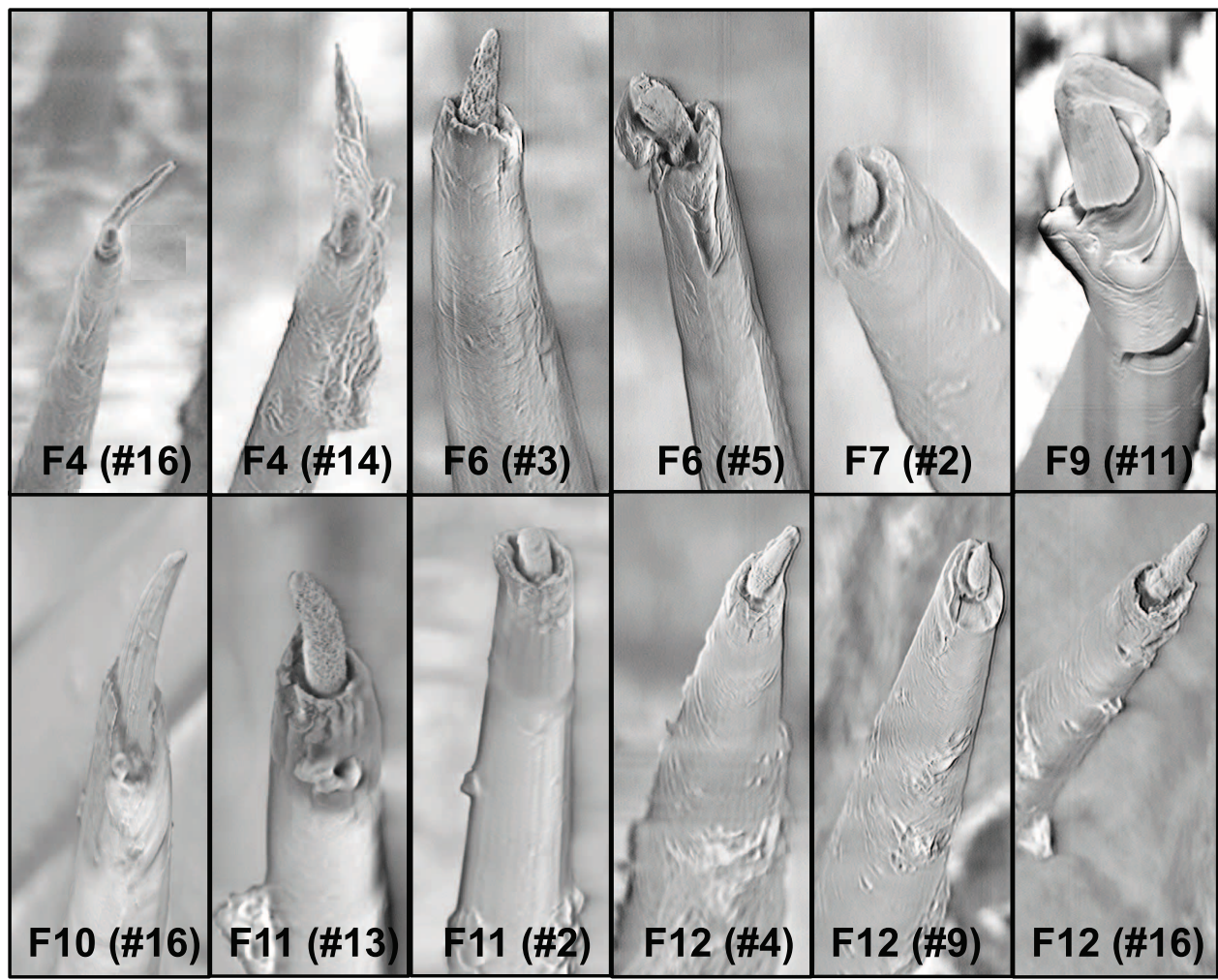

FIGURE 4 | (A) Pre-implant and post-explant SEM. Pre-implant and post-explant SEM images to show minimal to moderate changes at the recording site structure for the longest term animals. However, recording site changes occur as a result of corrosion of the recording surface for long-term implants. Shown here are three 6-month implants (F2, F4, F6), two 3-month animals (F7, F8), one 15-day animal (F11), and one 7-day animal (F14), respectively. A crack at the recording site can be observed to be developing in the electrode F2 (no. 5). (B) Insulation deterioration post-explant SEM. Post-explant SEM of individual microwire to indicate deterioration in electrode insulation for parylene-C coated $\mathrm{Pt} / \mathrm{Ir}$ microwires. The deterioration occurs in the form of delamination and cracks. While the insulation deterioration varies among microwires even with the same array, we observed it to be present in all the wires across animals for all implant durations (7 days -6 months) 


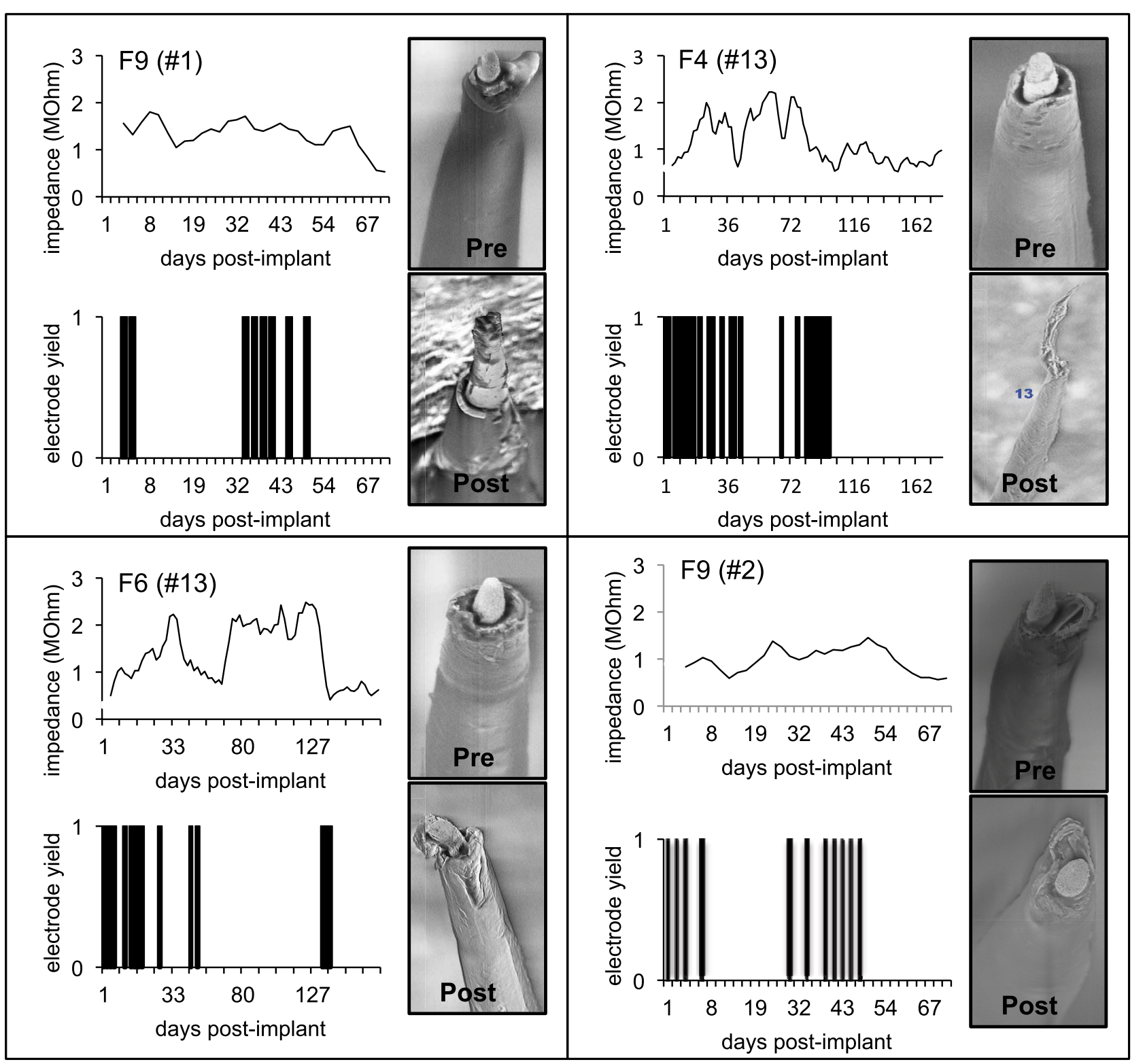

FIGURE 5 | Effect of insulation damage. Pre-implant and post-explant SEM images are shown for four Pt/Ir electrodes in three chronic animals that undergoes delamination during their respective implant durations. Insulation delamination results in a decline in $1 \mathrm{KHz}$ electrode impedance combined with poor functional performance (low yield). Functional performance is characterized by electrode yield on each of the recording days. A zero yield suggests that the respective electrode was not able to record a single unit whereas a 1 suggests that the electrode was able to record a single unit. histopathology markers which is consistent with our hypothesis that neuroinflammation was not correlated with electrode performance.

\section{DISCUSSION}

We presented in this study an in vivo abiotic and biotic characterization approach for $\mathrm{Pt} / \mathrm{Ir}$ microelectrode arrays in chronic implants. The study combined SEM imaging, impedance spectroscopy, histopathology with electrode functional performance so that better understanding of long-term microelectrode performance can be achieved. Abiotic and biotic measurements were performed over 6-months of implant duration revealing changes that occurred at the electrode-tissue interface affecting functional performance of the microelectrodes. This study was predicated on the hypothesis that failure of this electrode type occurred as a result of both abiotic and biotic factors. Pre-implant SEM imaging showed significant variation between electrodes even within the same array indicating manufacturing imperfections. Post-explant SEM imaging indicated that $\mathrm{Pt} / \mathrm{Ir}$ electrodes did not suffer from deterioration due to corrosion 


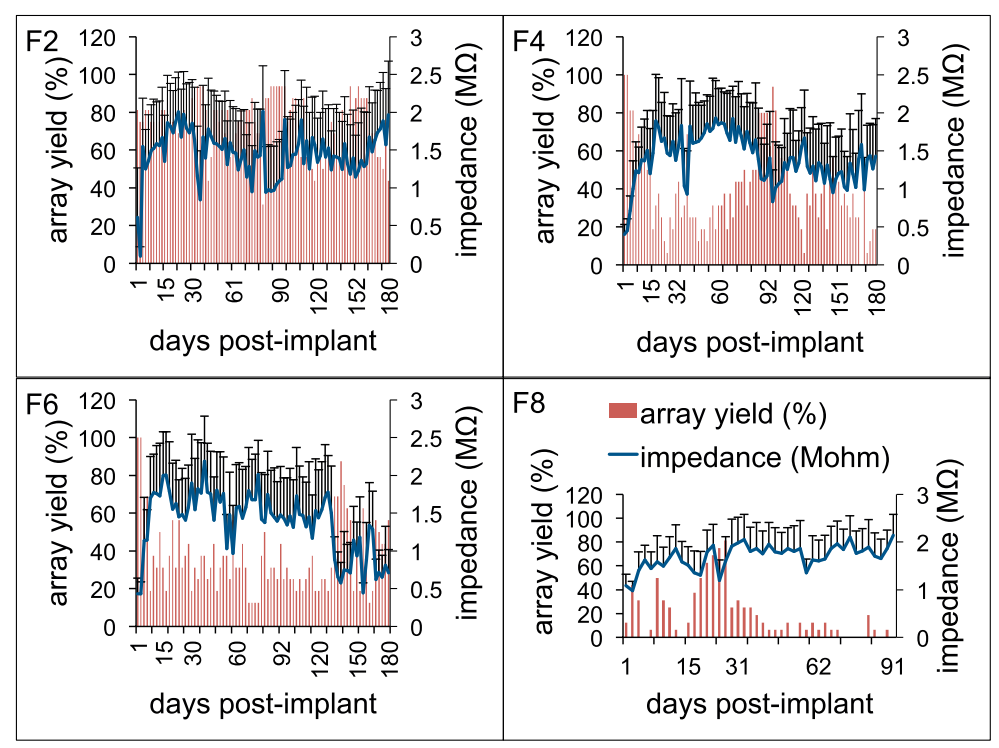

FIGURE 6 | Electrode impedance and electrophysiology trends. Daily array yield (red) and average $1 \mathrm{KHz}$ impedance trends (blue) for four long-term animals (F2, F4, F6: 6-months; F8: 3-months) to show the large daily variations in electrode impedance that result in low array yield. Array yield was defined as the percentage of electrodes out of 16 electrodes in an array that was able to isolate at least a single neuron during a recording session. Error bars depict standard deviations among the impedance values recorded from 16 electrodes during a recording session. like tungsten (Prasad et al., 2012), however electrodes exhibited deformation of the recording sites such as bent or cracked tips. In addition, deterioration of insulation material was observed among all electrodes in all animals in the form of insulation delamination and cracking. Generally, electrode impedance reduced chronically in electrodes that had insulation deterioration and poor functional performance. Histopathological evaluation suggested modest elevation of microglial markers for longest-term animals. In contrast, we show evidence in this study that the insulation deterioration, manufacturing, and corrosion were the likely abiotic factors contributing to the electrode failure. In addition to the chronic abiotic and biotic effects, we provide evidence that vascular disruption and concurrent intraparenchymal hemorrhaging during brain implantation is a major biotic factor that likely contributes to electrode failure.

The results of this study indicate a need for precision and consistency of manufacturing of microelectrode arrays, an improvement that will likely play a major role in the long-term performance (Prasad and Sanchez, 2012; Prasad et al., 2012; Streit et al., 2012; Barrese et al., 2013). Of particular interest is the recording site itself and interface between that site and the surrounding insulation. Pre-implant variations such as those depicted in Figure 3 provide a weak or fault site on the microelectrodes that will lead to an accelerated deterioration of the recording surface of individual electrodes. We observed that electrodes with imperfections prior to implantation were more susceptible to morphological changes during the implant period (Figure 5). Manufacturing defects at the recording sites provided fault sites where accelerated deterioration happened resulting in a decreased electrode impedance and poor functional performance
(Figure 5). Therefore, to mitigate the variability arising out of the manufacturing process, greater quality control measures should be employed pre-implant to ensure that implanted electrodes are all of good quality. The manufacturer of the FMAs manipulated the amount of deinsulation for each microwire during the laser cutting process to control the impedance. For this study, FMAs were custom-ordered with $100-150 \mathrm{~K} \Omega$ as the impedance for each microwire. That value of impedance was chosen based upon our experience with tungsten microwires that had the best electrode yield for the impedance range 40-150 K $\Omega$ (Prasad and Sanchez, 2012). Pre-implant baseline impedance measurements for these Pt/Ir FMAs were in the range $100-400 \mathrm{~K} \Omega$ at $1 \mathrm{kHz}$ measured in PBS. While the intent of the deinsulation process was to control impedance, observations from the electrodes used in this study revealed that the process is not as precise as would be desired and there is inconsistency in the manufacturing process (Figure 3). Variance in the starting impedance and manufacturing is compounded by changes in impedance following surgery, which were approximately 2-3 times compared to that measured in vitro. The impedance increased progressively over the next 4week period reaching up to 2-3 $\mathrm{M} \Omega$. We observed larger increase in impedance value for these microwires in the first 2-3 weeks as compared to tungsten microwires during the same time period (Williams et al., 2007; Ward et al., 2009; Prasad et al., 2012). While in general, impedance value for tungsten microwires was in the range 30-400 K $\Omega$ (Prasad and Sanchez, 2012), the impedance of $\mathrm{Pt} / \mathrm{Ir}$ microwires were in the order of 1-3 M $\Omega$ for most electrodes during the course of chronic implant. This may be attributed to the sharp recording surfaces (smaller geometric surface area) of the $\mathrm{Pt} / \mathrm{Ir}$ microwires compared to blunt cut recording tips (larger geometric surface area) of tungsten microwires. We and 

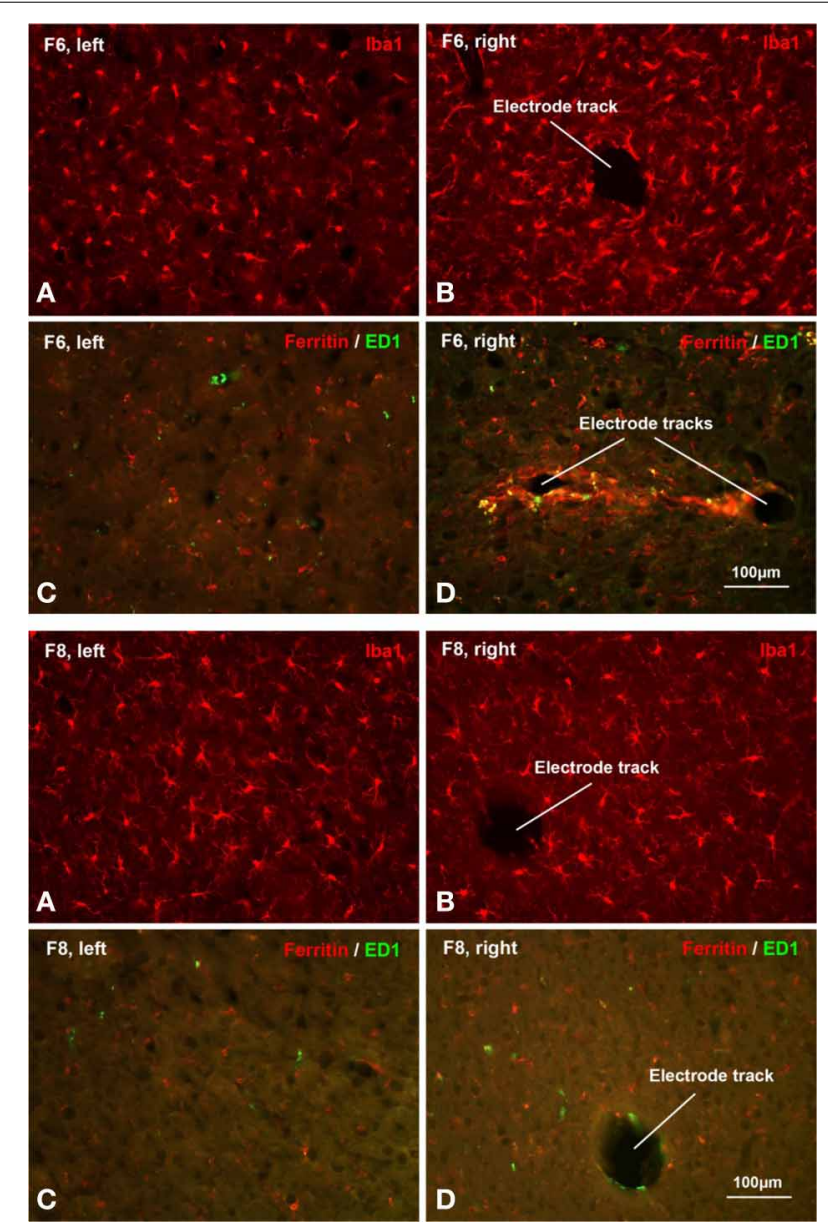

FIGURE 7 | Histopathology. Histopathological staining of microglial cells in two chronically implanted animals, F8 (91 days, top panel) and F6 (180 days, bottom panel). In F8, poor electrode array yield was correlated with minimal microglial activation. Comparing Iba1 labeling on the non-implanted contralateral side (A) with the implanted side (B) it is evident that microglia display a characteristic resting morphology as well as similar density on both sides. There was only modest upregulation of ED1 and ferritin expression in F8, as seen in Table 2 and in panels (C,D). In F6, a moderate-to-good array yield correlated with minimal microglial activation. Comparing panels $(\mathbf{A}, \mathbf{B})$ microglia appear slightly hypertrophic and somewhat increased in number. Ferritin expression was upregulated modestly and to a similar extent as in F8 (Table 2). Panel (D) shows an example of the maximal ferritin upregulation observed around electrode tracks in this animal.

others have reported for tungsten microwires large daily variation in impedance values within an array during the first 3-4 weeks after which the impedance reduced and there were smaller daily variations in the chronic period (Ward et al., 2009; Prasad and Sanchez, 2012; Prasad et al., 2012). In comparison, there was greater day-to-day variability in impedances for $\mathrm{Pt} / \mathrm{Ir}$ microwires during the entire implant duration as suggested by large error bars (Figure 6). In addition to the abiotic factors affecting electrode impedance, the trends in impedance during the chronic lifetime of the electrodes also suggest the involvement of biotic factors. The cellular changes occurring in the local environment of the
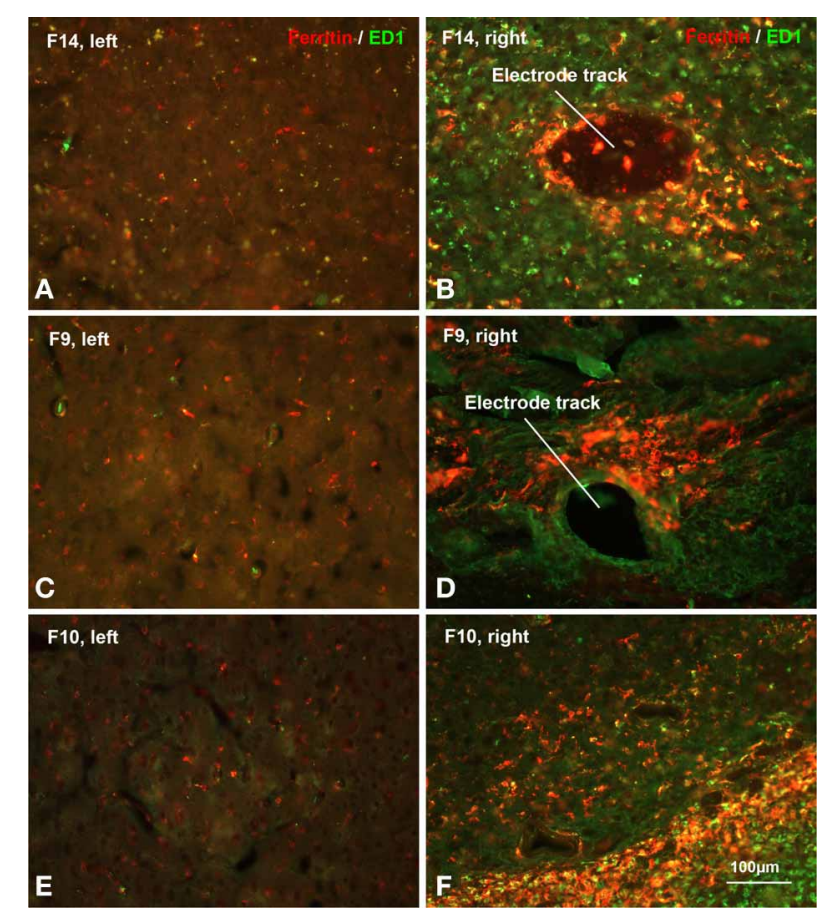

FIGURE 8 | Hemorrhage. Increased ferritin expression in animals with poor electrode performance. Shown are micrographs from three different rats all illustrating dramatic upregulation of ferritin (see also Table 2). (A,B) F14 is 7 dpi (days post-implantation) and shows a 20-fold increase; (C,D) F9 is $71 \mathrm{dp}$ and shows a 48-fold increase; (E,F) F10 is $21 \mathrm{dpi}$ and shows a 91-fold increase in ferritin expression over the control (left) side. The animals also show strong upregulation of both Iba1 and ED1 markers (Table 2). These histopathological findings are indicative of significant tissue damage, hemorrhage, and BBB disruption.

electrode tips is a likely contributor of the daily changes in the observed electrode impedance (Williams et al., 2007).

In addition to the electrode insulation interface, our results strongly suggest the bulk insulation itself is an important contributor leading to electrode failure. We have evaluated two of the most commonly used insulation materials in neural electrodes: Polyimide coated tungsten wires (Prasad et al., 2012) and Parylene-C coated $\mathrm{Pt} / \mathrm{Ir}$ wires (in this study). Insulation delamination and cracking was evident among all wires across all animals for both insulation types (Figure 4B). However, there was a large variation in the extent of the insulation deterioration even between electrodes in the same array. We observed electrodes that had insulation damage pre-implant were more prone to undergo insulation deterioration during the chronic implant (Figure 5). Insulation damage results in leakage resistance and parasitic capacitance (Kane et al., 2013) and continuous decrease in impedance, which leads to an accelerated damage causing decrease in electrode functional performance (Figure 5). These results indicate that electrode delamination and cracking to be a significant failure mode and steps must be taken in order to evaluate better electrode insulation materials in future.

Material differences should also be considered while choosing electrode metals for recording purposes. In previous studies, we 
Table 2 | Histopathology.

\begin{tabular}{|c|c|c|c|c|c|c|c|c|}
\hline Animal groups & Rat No. & Days post-implant & \multicolumn{2}{|c|}{ lba1 } & \multicolumn{2}{|c|}{ ED1 } & \multicolumn{2}{|c|}{ Ferritin } \\
\hline & F12 & 7 & 2226 & 14,248 & 74 & 998 & 745 & 18,330 \\
\hline & F13 & 15 & 4219 & 14,747 & 78 & 687 & 288 & 2685 \\
\hline & F14 & 7 & 1731 & 15,941 & 131 & 881 & 248 & 4974 \\
\hline \multirow[t]{5}{*}{ Chronic implants ( $\leq 6$ months) } & F9 & 71 & 850 & 16,137 & 16 & 253 & 64 & 3094 \\
\hline & F10 & 21 & 1555 & 15,149 & 59 & 1157 & 78 & 7106 \\
\hline & $\mathrm{F} 2$ & 180 & 3519 & 4133 & 594 & 280 & 911 & 2273 \\
\hline & $\mathrm{F} 4$ & 180 & 2402 & 3158 & 260 & 385 & 976 & 2481 \\
\hline & F6 & 180 & 1836 & 2647 & 125 & 125 & 950 & 2839 \\
\hline
\end{tabular}

Total positive areas of each of the stains for all animals with Pt/lr array implants. Right refers to the implanted tissue and left refers to the contralateral control tissue.

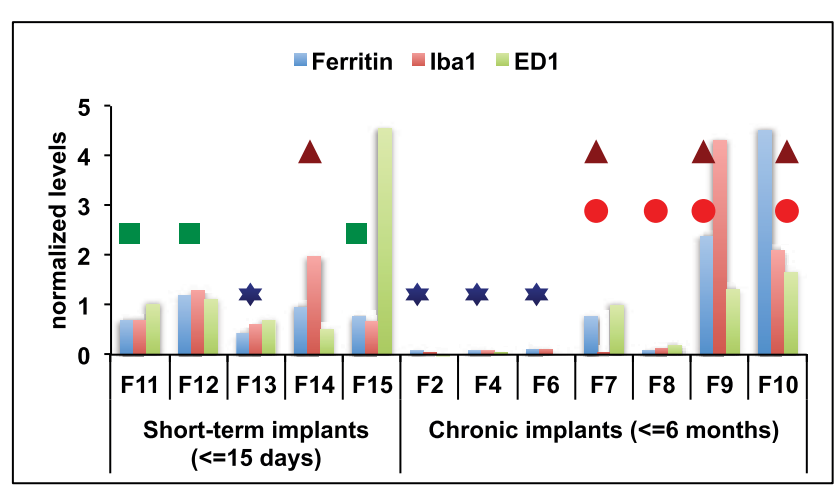

Moderate-to-good functional

performance (average yield $>35 \%$ ) with low ferritin levels

Poor functional performance, pre-implant imperfections and post-explant gross recording site changes
Good performance, high histopathology marker levels

Aigh ferritin levels, poor performance

FIGURE 9 | Combined analysis. Combined abiotic-biotic analysis to include histological, morphological, and functional performance for all animals to indicate contributing failure modes.

have observed that tungsten undergoes rapid corrosion in vitro (Patrick et al., 2011) and in vivo for implants as early as 1-week following surgery (Prasad et al., 2012). We reported that deterioration of tungsten continued in the chronic phase in the form of recording sites becoming more recessed. Therefore, choice of recording material was one of the contributing abiotic failure modes for tungsten electrodes. However, we observed in this study that $\mathrm{Pt} / \mathrm{Ir}$ provides better recording surfaces and corrosion was not as big of an issue as with tungsten. Figure 4A shows various $\mathrm{Pt} / \mathrm{Ir}$ electrodes both pre-implant and post-explant for varying implant durations ( 7 days -6 months). We can observe that corrosion was not a significant problem. We did not observe recessed recording sites like tungsten in any of the electrodes and across animals. Even for electrodes, which underwent large morphological changes at the electrode recording sites (Figure 4B), electrodes were not as much affected by corrosion. Therefore, corrosion does not appear to be a significant contributing factor to electrode failure for $\mathrm{Pt} / \mathrm{Ir}$ electrodes.

One of the experimental design factors that enabled deeper insight to electrode failure is that we coupled electrode array functional performance with impedance spectroscopy to study if there was a functional relationship between them. Electrode 
array functional performance was quantified by array yield which was defined as the percentage of electrodes in an array that were able to isolate single units. Higher array yield are desirable for a neuroprosthetic application. In general for these floating arrays, $1 \mathrm{kHz}$ impedance magnitude trends were similar for long-term animals where impedance increased progressively in the first 2-3 weeks and then decreased in the subsequent weeks with occasional increases and decreases in the chronic period (Figure 6). We observed that array yield generally declined during this initial 2-3 week period following implant attributed as a result of surgical trauma in other studies (Biran et al., 2005). Poor array yield ( $<25 \%$ yield) was observed when impedance was high $(>1.5 \mathrm{M} \Omega)$ and yield increased when impedance was low during the late chronic period (>12 weeks) for long-term animals (Figure 6). The daily variations observed in average impedance magnitude corresponded with moderate $(25-70 \%$ yield $)$ to poor $(<25 \%$ yield $)$ functional yield from these $\mathrm{Pt} / \mathrm{Ir}$ MEAs among all animals (Table 1).

There is a cascade of biological reactions that occur following an electrode implant surgery that in brief includes activation and recruitment of astrocytes, microglia, and macrophages at the implant site in order to isolate the implanted device and repair the damaged tissue (Polikov et al., 2005; Thelin et al., 2011; Kozai et al., 2012b). Several studies have pointed out the time course of such biological reactions occurring at the tissue-electrode interface that begin as early as right after insertion and continue as long as the implant is in the neural tissue (Szarowski et al., 2003; Freire et al., 2011; Prasad et al., 2012). Of course, the most obvious and inevitable biological consequence of intracerebral electrode insertion, or for that matter any type of traumatic brain injury, is a neuroinflammatory response. Neuroinflammation is mediated primarily by activated microglial cells which is why we have focused our attention on these cells in this and prior studies (Prasad et al., 2011, 2012; Streit et al., 2012). Our findings with regard to activated microglia were as expected in that electrode implantation elicited widespread microglial activation at the implantation site, which in most cases became somewhat attenuated over time. However, we also studied microglial degeneration, evident as cytoplasmic fragmentation, which we believe is a direct consequence of oxidative stress brought about by influx of free iron through intracerebral bleeding. In contrast to our prior study with tungsten microwires where microglial degeneration was observed only during early survival times, the current study shows that microglial degeneration can also occur during relatively late time points (Figure 8), especially in animals where there was significant intracerebral bleeding thus supporting our belief that microglial cytoplasmic fragmentation occurs as a direct consequence of iron-mediated oxidative stress.

In conclusion, the results of our study serve to further illuminate the problem of electrode failure by focusing attention on two major failure modes, one abiotic and the other one biotic. Future efforts geared toward prolonging the functional lifetime of electrodes should thus be focused on further improving electrode manufacturing practices and on innovative ways of minimizing intracerebral bleeding and associated oxidative stress.

\section{ACKNOWLEDGMENTS}

This work was sponsored by the Defense Advanced Research Projects Agency (DARPA) Microsystems Technology Office under the auspices of Dr. Jack Judy (jack.judy@darpa.mil) through the Space and Naval Warfare Systems Center, Pacific Grant No. N66001-11-1-4009.

\section{REFERENCES}

Andrei, A., Welkenhuysen, M., Nuttin, B., and Eberle, W. (2012). A response surface model predicting the in vivo insertion behavior of micromachined neural implants. J. Neural Eng. 9:016005. doi: 10.1088/1741-2560/9/1/016005

Andrews, R. J. (2010). Neuromodulation: advances in the next five years. Ann. N.Y. Acad. Sci. 1199, 204-211. doi: 10.1111/j.1749-6632.2009.05379.x

Barrese, J. C., Rao, N., Paroo, K., Triebwasser, C., Vargas-Irwin, C., Franquemont, L., et al. (2013). Failure mode analysis of silicon-based intracortical microelectrode arrays in non-human primates. J. Neural Eng. 10:066014. doi: 10.1088/ 1741-2560/10/6/066014

Biran, R., Martin, D. C., and Tresco, P. A. (2005). Neuronal cell loss accompanies the brain tissue response to chronically implanted silicon microelectrode arrays. Exp. Neurol. 195, 115-126. doi: 10.1016/j.expneurol.2005.04.020

Biran, R., Martin, D. C., and Tresco, P. A. (2007). The brain tissue response to implanted silicon microelectrode arrays is increased when the device is tethered to the skull. J. Biomed. Mater. Res. 82, 169-178. doi: 10.1002/jbm.a.31138

Brett, M. A. C., and Brett, A. M. O. (1993). Electrochemistry: Principles, Methods and Applications. New York, NY: Oxford Science.

Cogan, S. F. (2008). Neural stimulation and recording electrodes. Annu. Rev. Biomed. Eng. 10, 275-309. doi: 10.1146/annurev.bioeng.10.061807.160518

Collinger, J. L., Wodlinger, B., Downey, J. E., Wang, W., Tyler-Kabara, E. C., Weber, D. J., et al. (2013). High-performance neuroprosthetic control by an individual with tetraplegia. Lancet 381, 557-564. doi: 10.1016/S0140-6736(12) 61816-9

Csicsvari, J., Henze, D. A., Jamieson, B., Harris, K. D., Sirota, A., Barthó, P., et al. (2003). Massively parallel recording of unit and local field potentials with silicon-based electrodes. J. Neurophysiol. 90, 1314-1323. doi: 10.1152/jn.00116.2003

Dijkstra, C. D., Dopp, E. A., Joling, P., and Kraal, G. (1985). The Heterogeneity of mononuclear phagocytes in lymphoid organs - distinct macrophage subpopulations in the rat recognized by monoclonal antibody-Ed1, antibody-Ed 2 and antibody-Ed3. Immunology 54, 589-599.

Drake, K. L., Wise, K. D., Farraye, J., Anderson, D. J., and Bement, S. L. (1988). Performance of planar multisite microprobes in recording extracellular single-unit intracortical activity. IEEE Trans. Biomed. Eng. 35, 719-732. doi: $10.1109 / 10.7273$

Edell, D. J., Vo Van, T., McNeil, V. M., and Clark, L. D. (1992). Factors influencing the biocompatibility of insertable silicon microshafts in cerebral cortex. IEEE Trans. Biomed. Eng. 39, 635-643. doi: 10.1109/10.141202

Ethier, C., Oby, E. R., Bauman, M. J., and Miller, L. E. (2012). Restoration of grasp following paralysis through brain-controlled stimulation of muscles. Nature 485, 368-371. doi: 10.1038/nature 10987

Frampton, J. P., Hynd, M. R., Shuler, M. L., and Shain, W. (2010). Effects of glial cells on electrode impedance recorded from neuralprosthetic devices in vitro. Ann. Biomed. Eng. 38, 1031-1047. doi: 10.1007/s10439-010-9911-y

Freire, M. A., Morya, E., Faber, J., Santos, J. R., Guimaraes, J. S., Lemos, N. A., et al. (2011). Comprehensive analysis of tissue preservation and recording quality from chronic multielectrode implants. PLOS ONE 6:e27554. doi: 10.1371/journal.pone.0027554

Geddes, L. A. (1997). Historical evolution of circuit models for the electrodeelectrolyte interface. Ann. Biomed. Eng. 25, 1-14. doi: 10.1007/BF02738534

Geddes, L. A., and Roeder, R. (2003). Criteria for the selection of materials for implanted electrodes. Ann. Biomed. Eng. 31, 879-890. doi: 10.1114/1.1581292

Grill, W. M., and Mortimer, J. T. (1994). Electrical properties of implant encapsulation tissue. Ann. Biomed. Eng. 22, 23-33. doi: 10.1007/BF02368219

Hochberg, L. R., Bacher, D., Jarosiewicz, B., Masse, N. Y., Simeral, J. D., Vogel, J., et al. (2012). Reach and grasp by people with tetraplegia using a neurally controlled robotic arm. Nature 485, 372-375. doi: 10.1038/nature11076

Iosa, M., Morone, G., Fusco, A., Bragoni, M., Coiro, P., Multari, M., et al. (2012). Seven capital devices for the future of stroke rehabilitation. Stroke Res. Treat. 2012:187965. doi: 10.1155/2012/187965 
Jackson, A., and Fetz, E. E. (2007). Compact movable microwire array for longterm chronic unit recording in cerebral cortex of primates. J. Neurophysiol. 98, 3109-3118. doi: 10.1152/jn.00569.2007

Jackson, A., and Zimmermann, J. B. (2012). Neural interfaces for the brain and spinal cord-restoring motor function. Nat. Rev. Neurol. 8, 690-699. doi: 10.1038/nrneurol.2012.219

Johnson, M. D., Otto, K. J., and Kipke, D. R. (2005). Repeated voltage biasing improves unit recordings by reducing resistive tissue impedances. IEEE Trans. Neural Syst. Rehabil. Eng. 13, 160-165. doi: 10.1109/TNSRE.2005.847373

Kam, L., Shain, W., Turner, J. N., and Bizios, R. (1999). Correlation of astroglial cell function on micro-patterned surfaces with specific geometric parameters. Biomaterials 20, 2343-2350. doi: 10.1016/S0142-9612(99)00163-5

Kane, S. R., Cogan, S. F., Ehrlich, J., Plante, T. D., McCreery, D. B., and Troyk, P. R. (2013). Electrical performance of penetrating microelectrodes chronically implanted in cat cortex. IEEE Trans. Biomed. Eng. 60, 2153-2160. doi: 10.1109/ TBME.2013.2248152

Karumbaiah, L., Saxena, T., Carlson, D., Patil, K., Patkar, R., Gaupp, E. A., et al. (2013). Relationship between intracortical electrode design and chronic recording function. Biomaterials 34, 8061-8074. doi: 10.1016/j.biomaterials.2013. 07.016

Kim, B. J., Kuo, J. T., Hara, S. A., Lee, C. D., Yu, L., Gutierrez, C. A., et al. (2013). 3D Parylene sheath neural probe for chronic recordings. J. Neural Eng. 10:045002. doi: 10.1088/1741-2560/10/4/045002

Kipke, D. R., Vetter, R. J., Williams, J. C., and Hetke, J. F. (2003). Silicon-substrate intracortical microelectrode arrays for long-term recording of neuronal spike activity in cerebral cortex. IEEE Trans. Neural Syst. Rehabil. Eng. 11, 151-155. doi: 10.1109/TNSRE.2003.814443

Koivuniemi, A. S., and Otto, K. J. (2011). Asymmetric versus symmetric pulses for cortical microstimulation. IEEE Trans. Neural Syst. Rehabil. Eng. 19, 468-476. doi: 10.1109/TNSRE.2011.2166563

Kozai, T. D. Y., Langhals, N. B., Patel, P. R., Deng, X. P., Zhang, H. N., Smith, K. L., et al. (2012a). Ultrasmall implantable composite microelectrodes with bioactive surfaces for chronic neural interfaces. Nat. Mater. 11, 1065-1073. doi: $10.1038 /$ nmat3468

Kozai, T. D., Vazquez, A. L., Weaver, C. L., Kim, S. G., and Cui, X. T. (2012b). In vivo two-photon microscopy reveals immediate microglial reaction to implantation of microelectrode through extension of processes. J. Neural Eng. 9:066001. doi: 10.1088/1741-2560/9/6/066001

Lee, H., Bellamkonda, R. V., Sun, W., and Levenston, M. E. (2005). Biomechanical analysis of silicon microelectrode-induced strain in the brain. J. Neural Eng. 2, 81-89. doi: 10.1088/1741-2560/2/4/003

Lempka, S. F., Johnson, M. D., Moffitt, M. A., Otto, K. J., Kipke, D. R., and McIntyre, C. C. (2011). Theoretical analysis of intracortical microelectrode recordings. J. Neural Eng. 8:045006. doi: 10.1088/1741-2560/8/4/045006

Lopes, K. O., Sparks, D. L., and Streit, W. J. (2008). Microglial dystrophy in the aged and Alzheimer's disease brain is associated with ferritin immunoreactivity. Glia 56, 1048-1060. doi: 10.1002/glia.20678

Ludwig, K. A., Langhals, N. B., Joseph, M. D., Richardson-Burns, S. M., Hendricks, J. L., and Kipke, D. R. (2011). Poly(3,4-ethylenedioxythiophene) (PEDOT) polymer coatings facilitate smaller neural recording electrodes. J. Neural Eng. 8:014001. doi: 10.1088/1741-2560/8/1/014001

Ludwig, K. A., Uram, J. D., Yang, J., Martin, D. C., and Kipke, D. R. (2006) Chronic neural recordings using silicon microelectrode arrays electrochemically deposited with a poly(3,4-ethylenedioxythiophene) (PEDOT) film. J. Neural Eng. 3, 59-70. doi: 10.1088/1741-2560/3/1/007

Maling, N., Hashemiyoon, R., Foote, K. D., Okun, M. S., and Sanchez, J. C. (2012). Increased thalamic gamma band activity correlates with symptom relief following deep brain stimulation in humans with tourette's syndrome. PLoS ONE 7:e44215. doi: 10.1371/journal.pone.0044215

McConnell, G. C., Butera, R. J., and Bellamkonda, R. V. (2009a). Bioimpedance modeling to monitor astrocytic response to chronically implanted electrodes. J. Neural Eng. 6:055005. doi: 10.1088/1741-2560/6/5/055005

McConnell, G. C., Rees, H. D., Levey, A. I., Gutekunst, C. A., Gross, R. E., and Bellamkonda, R. V. (2009b). Implanted neural electrodes cause chronic, local inflammation that is correlated with local neurodegeneration. J. Neural Eng. 6:056003. doi: 10.1088/1741-2560/6/5/056003

Merrill, D. R., and Tresco, P. A. (2005). Impedance characterization of microarray recording electrodes in vitro. IEEE Trans. Biomed. Eng. 52, 1960-1965. doi: 10.1109/TBME.2005.856245
Mollazadeh, M., Aggarwal, V., Davidson, A. G., Law, A. J., Thakor, N. V., and Schieber, M. H. (2011). Spatiotemporal Variation of Multiple Neurophysiological Signals in the Primary Motor Cortex during Dexterous Reach-to-Grasp Movements. J. Neurosci. 31, 15531-15543. doi: 10.1523/ JNEUROSCI.2999-11.2011

Morrell, M. J. (2011). Responsive cortical stimulation for the treatment of medically intractable partial epilepsy. Neurology 77, 1295-1304. doi: 10.1212/WNL.0b013e3182302056

Musallam, S., Bak, M. J., Troyk, P. R., and Andersen, R. A. (2007). A floating metal microelectrode array for chronic implantation. J. Neurosci. Methods 160, 122-127. doi: 10.1016/j.jneumeth.2006.09.005

Nicolelis, M. A. L. (1999). Methods for Neural Ensemble Recordings. Boca Raton, FL: CRC Press.

Nicolelis, M. A. L., Dimitrov, D., Carmena, J. M., Crist, R., Lehew, G., Kralik, J. D., et al. (2003). Chronic, multisite, multielectrode recordings in macaque monkeys. Proc. Natl. Acad. Sci. U.S.A. 100, 11041-11046. doi: 10.1073/pnas.1934665100

Otto, K. J., Johnson, M. D., and Kipke, D. R. (2006). Voltage pulses change neural interface properties and improve unit recordings with chronically implanted microelectrodes. IEEE Trans. Biomed. Eng. 53, 333-340. doi: 10.1109/TBME.2005.862530

Otto, K. J., Rousche, P. J., and Kipke, D. R. (2005). Cortical microstimulation in auditory cortex of rat elicits best-frequency dependent behaviors. J. Neural Eng. 2, 42-51. doi: 10.1088/1741-2560/2/2/005

Patrick, E., Orazem, M. E., Sanchez, J. C., and Nishida, T. (2011). Corrosion of tungsten microelectrodes used in neural recording applications. J. Neurosci. Methods 198, 158-171. doi: 10.1016/j.jneumeth.2011.03.012

Patrick, E., Ordonez, M., Alba, N., Sanchez, J. C., and Nishida, T. (2006). Design and fabrication of a flexible substrate microelectrode array for brain machine interfaces. Conf. Proc. IEEE Eng. Med. Biol. Soc. 1, 2966-2969. doi: 10.1109/ IEMBS.2006.260581

Polikov, V. S., Tresco, P. A., and Reichert, W. M. (2005). Response of brain tissue to chronically implanted neural electrodes. J. Neurosci. Methods 148, 1-18. doi: 10.1016/j.jneumeth.2005.08.015

Prasad, A., and Sanchez, J. C. (2012). Quantifying long-term microelectrode array functionality using chronic in vivo impedance testing. J. Neural Eng. 9:026028. doi: 10.1088/1741-2560/9/2/026028

Prasad, A., Sankar, V., Dyer, A. T., Knott, E., Xue, Q. S., Nishida, T., et al. (2011). Coupling biotic and abiotic metrics to create a testbed for predicting neural electrode performance. Conf. Proc. IEEE Eng. Med. Biol. Soc. 2011, 3020-3023. doi: 10.1109/IEMBS.2011.6090827

Prasad, A., Xue, Q. S., Sankar, V., Nishida, T., Shaw, G., Streit, W. J., et al. (2012). Comprehensive characterization and failure modes of tungsten microwire arrays in chronic neural implants. J. Neural Eng. 9:056015. doi: 10.1088/17412560/9/5/056015

Richter, A., Xie, Y., Schumacher, A., Loffler, S., Kirch, R. D., Al-Hasani, J., et al. (2013). A simple implantation method for flexible, multisite microelectrodes into rat brains. Front. Neuroeng. 6:6. doi: 10.3389/fneng.2013.00006

Rousche, P. J., and Normann, R. A. (1998). Chronic recording capability of the utah intracortical electrode array in cat sensory cortex. J. Neurosci. Methods 82, 1-15. doi: 10.1016/S0165-0270(98)00031-4

Rousche, P. J., Pellinen, D. S., Pivin, D. P. Jr., Williams, J. C., Vetter, R. J., and Kipke, D. R. (2001). Flexible polyimide-based intracortical electrode arrays with bioactive capability. IEEE Trans. Biomed. Eng. 48, 361-371. doi: 10.1109/ 10.914800

Rouse, A. G., Stanslaski, S. R., Cong, P., Jensen, R. M., Afshar, P., Ullestad, D., et al. (2011). A chronic generalized bi-directional brain-machine interface. J. Neural Eng. 8:036018. doi: 10.1088/1741-2560/8/3/036018

Sanchez, J. C., Alba, N., Nishida, T., Batich, C., and Carney, P. R. (2006). Structural modifications in chronic microwire electrodes for cortical neuroprosthetics: a case study. IEEE Trans. Neural Syst. Rehabil. Eng. 14, 217-221. doi: 10.1109/TNSRE.2006.875581

Saxena, T., Karumbaiah, L., Gaupp, E. A., Patkar, R., Patil, K., Betancur, M., et al. (2013). The impact of chronic blood-brain barrier breach on intracortical electrode function. Biomaterials 34, 4703-4713. doi: 10.1016/j.biomaterials.2013.03.007

Schmidt, E. M., Bak, M. J., and McIntosh, J. S. (1976). Long term chronic recording from cortical neurons. Exp. Neurol. 52, 496-506. doi: 10.1016/00144886(76)90220-X 
Stensaas, S. S., and Stensaas, L. J. (1978). Histopathological evaluation of materials implanted in the cerebral cortex. Acta Neuropathol. 41, 145-155. doi: 10.1007/BF00689766

Streit, W., Xue, Q. S., Prasad, A., Sankar, V., Knott, E., Dyer, A., et al. (2012). Electrode failure: tissue, electrical, and material responses. IEEE Pulse 3, 30-33. doi: 10.1109/MPUL.2011.2175632

Suner, S., Fellows, M. R., Vargas-Irwin, C., Nakata, G. K., and Donoghue, J. P. (2005). Reliability of signals from a chronically implanted, siliconbased electrode array in non-human primate primary motor cortex. IEEE Trans. Neural Syst. Rehabil. Eng. 13, 524-541. doi: 10.1109/TNSRE.2005. 857687

Szarowski, D. H., Andersen, M. D., Retterer, S., Spence, A. J., Isaacson, M., Craighead, H. G., et al. (2003). Brain responses to micro-machined silicon devices. Brain Res. 983, 23-35. doi: 10.1016/S0006-8993(03)03023-3

Thelin, J., Jorntell, H., Psouni, E., Garwicz, M., Schouenborg, J., Danielsen, N., et al. (2011). Implant size and fixation mode strongly influence tissue reactions in the CNS. PLoS ONE 6:e16267. doi: 10.1371/journal.pone.0016267

Truccolo, W., Donoghue, J. A., Hochberg, L. R., Eskandar, E. N., Madsen, J. R., Anderson, W. S., et al. (2011). Single-neuron dynamics in human focal epilepsy. Nat. Neurosci. 14, 635-641. doi: 10.1038/nn.2782

Turner, J. N., Shain, W., Szarowski, D. H., Andersen, M., Martins, S., Isaacson, M., et al. (1999). Cerebral astrocyte response to micromachined silicon implants. Exp. Neurol. 156, 33-49. doi: 10.1006/exnr.1998.6983

Vetter, R. J., Williams, J. C., Hetke, J. F., Nunamaker, E. A., and Kipke, D. R. (2004). Chronic neural recording using silicon-substrate microelectrode arrays implanted in cerebral cortex. IEEE Trans. Neural Syst. Rehabil. Eng. 51, 896-904. doi: 10.1109/TBME.2004.826680

Ward, M. P., Rajdev, P., Ellison, C., and Irazoqui, P. P. (2009). Toward a comparison of microelectrodes for acute and chronic recordings. Brain Res. 1282, 183-200. doi: 10.1016/j.brainres.2009.05.052
Williams, J. C., Hippensteel, J. A., Dilgen, J., Shain, W., and Kipke, D. R. (2007). Complex impedance spectroscopy for monitoring tissue responses to inserted neural implants. J. Neural Eng. 4, 410-423. doi: 10.1088/1741-2560/4/4/007

Williams, J. C., Rennaker, R. L., and Kipke, D. R. (1999a). Long-term neural recording characteristics of wire microelectrode arrays implanted in cerebral cortex. Brain Res. Protoc. 4, 303-313. doi: 10.1016/S1385-299X(99)00034-3

Williams, J. C., Rennaker, R. L., and Kipke, D. R. (1999b). Stability of chronic multichannel neural recordings: Implications for a long-term neural interface. Neurocomputing 26-27, 1069-1076. doi: 10.1016/S0925-2312(99)00106-X

Winslow, B. D., and Tresco, P. A. (2010). Quantitative analysis of the tissue response to chronically implanted microwire electrodes in rat cortex. Biomaterials 31, 1558-1567. doi: 10.1016/j.biomaterials.2009.11.049

Conflict of Interest Statement: The authors declare that the research was conducted in the absence of any commercial or financial relationships that could be construed as a potential conflict of interest.

Received: 09 December 2013; accepted: 14 January 2014; published online: 04 February 2014.

Citation: Prasad A, Xue Q-S, Dieme R, Sankar V, Mayrand RC, Nishida T, Streit WJ and Sanchez JC (2014) Abiotic-biotic characterization of Pt/Ir microelectrode arrays in chronic implants. Front. Neuroeng. 7:2. doi: 10.3389/fneng.2014.00002

This article was submitted to the journal Frontiers in Neuroengineering.

Copyright (c) 2014 Prasad, Xue, Dieme, Sankar, Mayrand, Nishida, Streit and Sanchez. This is an open-access article distributed under the terms of the Creative Commons Attribution License (CC BY). The use, distribution or reproduction in other forums is permitted, provided the original author(s) or licensor are credited and that the original publication in this journal is cited, in accordance with accepted academic practice. No use, distribution or reproduction is permitted which does not comply with these terms. 